\title{
Porous and Nanoporous Semiconductors and Emerging Applications
}

\author{
Helmut Föll, Jürgen Carstensen, and Stefan Frey \\ Chair for General Materials Science, Faculty of Engineering, Christian-Albrechts-University of Kiel, 24143 Kiel, Germany
}

Received 15 March 2006; Revised 14 September 2006; Accepted 3 October 2006

\begin{abstract}
Pores in single-crystalline semiconductors can be produced in a wide range of geometries and morphologies, including the "nanometer" regime. Porous semiconductors may have properties completely different from the bulk, and metamaterials with, for example, optical properties not encountered in natural materials are emerging. Possible applications of porous semiconductors include various novel sensors, but also more "exotic" uses as, for example, high explosives or electrodes for micro-fuel cells. The paper briefly reviews pore formation (including more applied aspects of large area etching), properties of porous semiconductors, and emerging applications.
\end{abstract}

Copyright (c) 2006 Helmut Föll et al. This is an open access article distributed under the Creative Commons Attribution License, which permits unrestricted use, distribution, and reproduction in any medium, provided the original work is properly cited.

\section{INTRODUCTION}

The dominant role of silicon in microelectronics along with the discovery of light-emitting nanoporous $\mathrm{Si}[1,2]$ has focused electrochemical investigations on this semiconductor even before 1991, and a large range of possible applications has emerged (for a recent review see [3]). While porous silicon was already discovered in 1957 by Uhlir [4] (but not recognized for what it was), it took until 2000 to find conditions for pore production in Ge [5-7]. Investigations of porous III-V semiconductors (mainly GaP, GaAs, InP) were also done more recently; see [8]. Pores in $\mathrm{GaN}[9]$, $\mathrm{SiC}[10,11]$, and $\mathrm{ZnSe}[12]$ have also been reported in the meantime, and the exploration of the available parameter space is an ongoing activity in many laboratories (cf. [13]).

\section{POROSIFICATION OF SEMICONDUCTORS}

It is helpful to first define a few terms to classify the tremendous variety of pores (cf. Figure 1). Pore geometry refers to (average) diameters of pores and distances between pores, that is, to the pore dimensions, while pore morphology addresses the pore shape (e.g., cylindrical, branched, facetted, fractal, ...).

According to IUPAC standards, micro-, meso-, or macropores refer to pores with typical dimensions of $<2 \mathrm{~nm}$, $2 \mathrm{~nm}-50 \mathrm{~nm}$, or $>50 \mathrm{~nm}$, respectively. The term "nanopores" thus is open to interpretation; here we use it somewhat loosely for pore dimensions well below $1 \mu \mathrm{m}$.
Generally, porosification can be obtained by anodizing the semiconductor in a suitable electrolyte, under suitable conditions. The necessary chemical reaction at the semiconductor-electrolyte interface is generally a mixture of direct dissolution, oxide formation, and oxide dissolution, with details sensitive to many parameters, for example, electrolyte chemistry, applied potential or current density, temperature, flow conditions of the electrolyte, doping type and level of the semiconductor, illumination state of the semiconductor (front or backside), and surface conditions (polished, rough, masked). The overall reaction may occur uniformly as electropolishing (often observed at high current densities) nonuniformly but everywhere on the surface, or highly localized including some self-organization-this is then the poreproducing mode.

While many details of the pore formation mechanism are still unclear, a few general statements can be made here. During pore formation, direct dissolution of the semiconductor almost always competes with oxidation plus subsequent dissolution of the oxide. The electrolyte therefore has to be able to dissolve the oxide. In the case of silicon, fluoridecontaining electrolytes are thus mandatory, while for other semiconductors the choice is more relaxed $\left(\mathrm{GeO}_{2}\right.$, e.g., even dissolves in water). A second prerequisite for the dissolution reaction and thereby pore formation in a semiconductor is electronic holes. In general, at least one hole is needed to initiate the reaction chain at the interface that ultimately leads to the "loss" of one atom (or molecule in the case of compound semiconductors). One of the major "tricks" for 


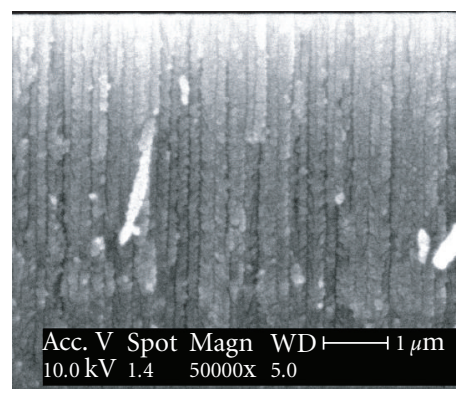

(a)

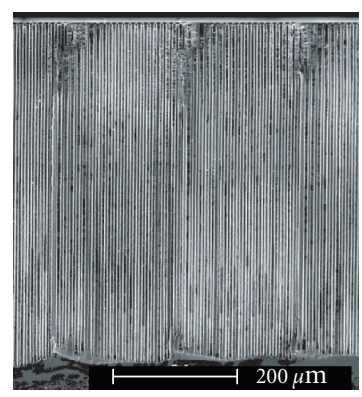

(b)

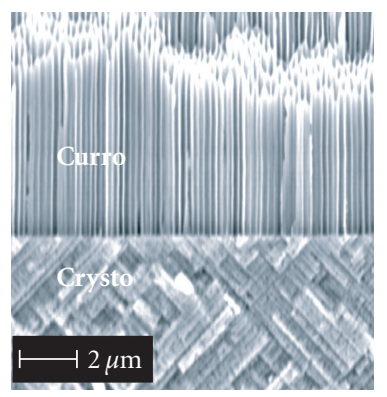

(c)

Figure 1: Variety of pores in semiconductors: (a) mesopores in Si, (b) macropores in Si with lithographic prestructuring, (c) "Curro"- “Crysto" transition in InP.

successful porosification experiments is to supply holes only locally. This trick obviously works only in $n$-doped material, where holes are the minority carriers. Holes then may be supplied by illumination [14], or via electrical tunneling or avalanche breakdown of the material due to high field strengths, as it is the case for the pores in III-V compounds [8]. However, while so far no pores could be obtained in most $p$-doped semiconductors, $\mathrm{Si}, \mathrm{SiC}[15]$, and very recently $\mathrm{Ge}$ $[7,16]$ provide the exceptions.

In the case of $p$-type materials it is necessary to drive the system in a state where holes can only be consumed at certain places. Generally, one operates in a regime with insufficient oxidation, either by a low current density ( $p$-type macropores in aqueous electrolytes [17]) or by using electrolytes that inherently produce worse and less oxide [3]. Alternatively, the use of a highly doped semiconductor, where most of the voltage is dropped on the electrolyte side of the junction, leads to a "fast" chemistry favoring direct dissolution in comparison to anodic oxidation ( $p$-type mesopores), again enhancing passivation relative to oxidation aspects during dissolution. Micropores in $p$-type Si are yet a different case; at least parts of their formation are due to quantum wire effects [2]. However, while a "quantum-wire model" can account for micropores in $\mathrm{Si}$, it is not specific to Si and thus fails to account for the absence of micropores in most other semiconductors up to now.

Pore formation in semiconductors is a highly interdisciplinary enterprise. There are even reasons to believe that the dissolution of the semiconductor electrode and all aspects of pore formation can only be sufficiently described by invoking in addition to all that was mentioned before, a stochastic component including self-organization in time and space, as championed in the "current burst" model [18].

\section{ETCHING AND ETCHING EQUIPMENT}

In order to obtain reproducible results, it is almost inevitable to use a potentiostat or a galvanostat with one or two reference electrodes instead of a simple power supply. While early experiments with illumination employed halogen lamps (with cold filters), high-intensity IR-LED arrays offer more flexibility and higher intensity for this kind of

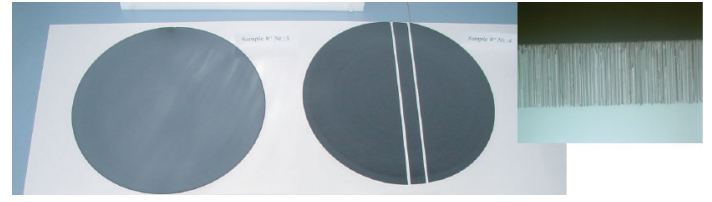

FIGURE 2: First electrochemically etched $200 \mathrm{~mm}$ p-type wafers (inset: macropores in organic electrolyte; the most demanding etching process).

experiments (for detailed information on all used hardware, see [19]). All chemical processes are strongly influenced by temperature, making it unavoidable to use computercontrolled cooling/heating systems with temperature stability in the $0.1^{\circ} \mathrm{C}$ region. To ensure constant electrolyte conditions at the interface, a rapid movement of the electrolyte is also unavoidable. Simple magneto-stirrers (or no stirring at all) will quickly lead to an insufficient uniformity in space and time. Considering that many electrolytes are highly dangerous and/or corrosive, these are demanding conditions even for small samples with areas in the $1 \mathrm{~cm}^{2}$ region, while the uniform etching of whole wafers becomes rather complex from a technical point of view.

To produce homogeneous porous layers over whole wafers a few setups have been proposed [3,20], which, however, address only parts of the parameter space. Our group has developed and commercialized [19] a large-area etcher meeting all requirements (including safety issues). The wafer is put on a vacuum chuck and hung head-over into the electrolyte. The electrolyte convection is ensured via a rocking table, on which the whole setup is mounted. Because the wafer and etching cell form an almost seamless surface, the rocking motion is enough to ensure sufficient and-on time average- $\mathrm{a}$ uniform electrolyte flow. This avoids potentially dangerous pumping equipment and allows an allTeflon design, which can accommodate even ultra-aggressive electrolytes, for example, HF/organic solvent mixtures which dissolve practically everything except Teflon. Wafers up to $200 \mathrm{~mm}$ diameter have been processed with superb uniformity (cf. Figure 2), and a scale-up to $300 \mathrm{~mm}$ is possible. 


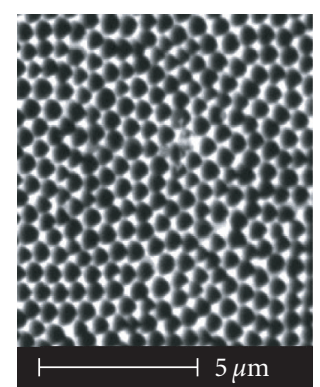

(a)

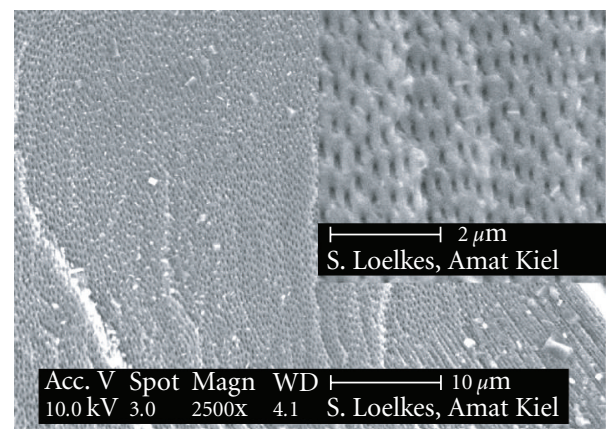

(d)

(b)
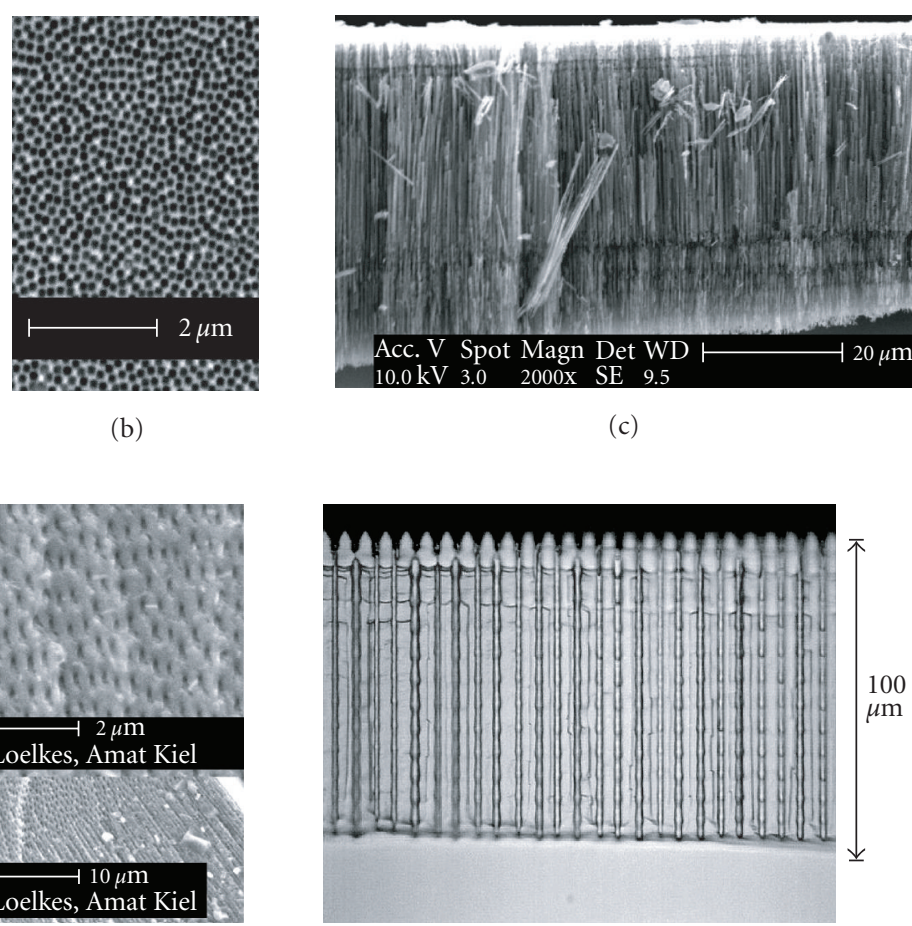

(e)

FIGURE 3: Fast pore etching in Si. (a) Plain view of pores on a (111) surface with hexagonal self-ordering. (b) On (100), a geometrically frustrated ordering of pores is evident. (c) Cross-section ((111) sample). (d) Homogeneous pore distribution of $100 \mathrm{~nm}$ pores in the crosssection of (111)-Si. (e) "Pulsed" fast macropore growth to a depth of $100 \mu \mathrm{m}$.

\section{MACRO-, MESO-, AND MICROPORES IN SILICON}

Macropores in Si were first discovered in 1990 [14], they are slowly growing (ca. $1 \mu \mathrm{m} / \mathrm{min}$ ) cylindrical pores with diameters between $350 \mathrm{~nm}$ and $10 \mu \mathrm{m}$ which are normally proceeding in $\langle 100\rangle$ and $\langle 113\rangle$ directions [3]. It is nearly impossible with other techniques to obtain such extreme aspect ratios. An inherent disadvantage of the standard macropore process ( $n$-Si under intense backside illumination) is the slow growth rate coupled to the necessarily low HF concentrations in this mode, which is a severe problem for any mass-production. However, our group has made some recent progress to boost the etching speed exploring two routes:

(i) coupling high HF-concentrations with a pulsed etching voltage while still employing backside illumination;

(ii) employing a less oxidizing organic electrolyte at high HF concentrations run in the breakdown mode without illumination [21].

In the first case the standard backside illumination setup is used, where a HF concentration higher than 6 wt.\% normally would lead to unacceptable bad pore morphologies (heavy branching, rough walls, etc.). This effect can be suppressed if the etching bias is periodically pulsed. While the pore diameter responds somewhat to the pulsing, the generation of side pores is largely suppressed with a drastic increase of etching speed (up to $4 \mu \mathrm{m} / \mathrm{min}$, cf. Figure $3(\mathrm{e})$ ).
In the second case it was attempted to mimic pore growth conditions as encountered in InP, where it is possible to generate beautiful pore arrays [22] with high etching rates. The hole generation in this case is confined to electrical breakdown, in contrast to standard macropore etching configuration, where breakdown is avoided because it generates small side pores which can perforate the whole pore walls (cf. .Figures 3, 5, and 6). The second ingredient predicted from an analysis of the InP pores was the suppression of oxide formation by using an organic solvent (acetonitrile $(\mathrm{MeCN})$ or dimethylformamide (DMF)) instead of water, supplemented by suitable salts because of the low conductivity of organic solvents. The growth speed in this case is then directly coupled to the oxide dissolution rate, as this process is by far the slowest process [18]. Therefore the HF concentration was pushed to $15-20 \mathrm{wt} \%$, under conditions which normal macropore growth does not proceed properly anymore.

First results were encouraging. Figure 3 shows some examples. As in InP, some self-organization was observed: on a (111) surface, where close packing induced ordering of pores and the natural threefold crystallographic symmetry harmonize, a high degree of hexagonal self-ordering was found, albeit not as pronounced as in the InP case. While on (100) InP, pores are still forming hexagonal arrays [22]; on (100) Si a completely new self-organization feature was found: the formation of a so-called "frustrated crystal." Since the fourfold symmetry of the crystal is competing with the hexagonal symmetry of close-packing, a geometrically frustrated 


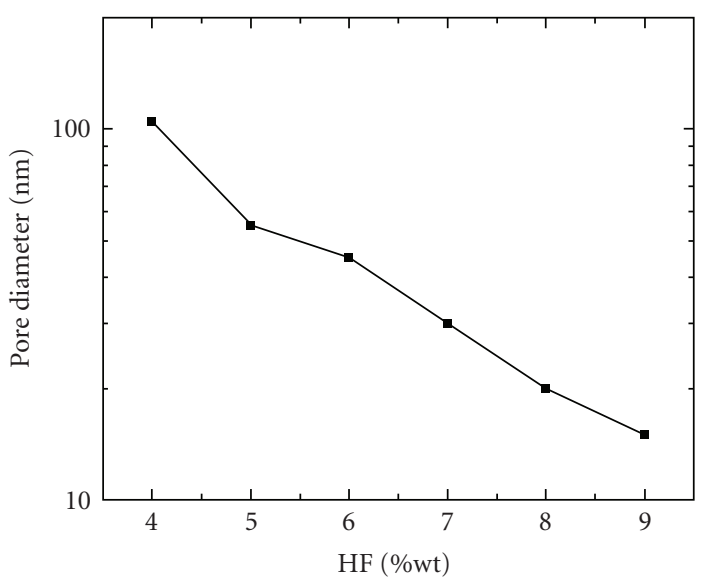

(a)

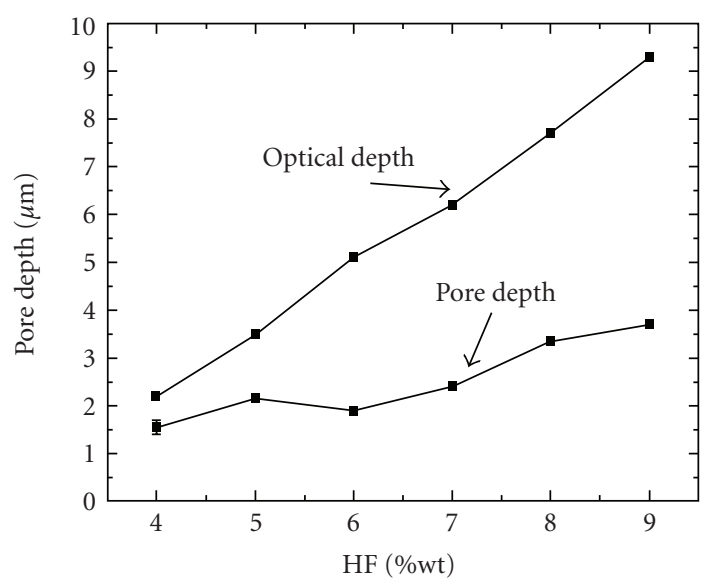

(b)

Figure 4: (a) Diameter and (b) depth of mesopores on $n$-Si $(100, \rho=5-20 \Omega \mathrm{cm}), j=30 \mathrm{~mA} / \mathrm{cm}^{2}, t_{\text {etch }}=100$ seconds. The "optical thickness" (ellipsometer) differs form the geometric thickness, because the pore density decreases, while the refractive index increases with increasing HF concentration.

distribution of pores results, with no angular correlation of nearest neighbor positions, but a strong correlation for second-nearest neighbors. This is a very appealing configuration for fundamental optic and magnetic investigations since it has a well-defined structure but without translational symmetry, which could, for example, cause unwanted Moirée patterns. It has to be stressed that these "macro" pores are not subject to the standard restrictions for macropores (i.e., growth speed $1 \mu \mathrm{m} / \mathrm{min}$, and diameter $>200 \mathrm{~nm}$ ) as can be seen from Figure 3, where several examples are shown with $100 \mathrm{~nm}$ pores; hence the regime of mesopores (following the strict IUPAC convention) is within reach.

Standard mesopores in Si are always grown on heavily doped samples and at relatively high HF concentrations, which necessitates only a few volts to drive large etching currents. The growth rates can reach several $10 \mu \mathrm{m} / \mathrm{min}$, which indicates that direct dissolution of silicon is the major process. The resulting morphology is a network of needle-like and heavily branched pores (cf. Figure 1(a)). Figure 4 shows the dependence of pore diameter and pore depth on fluoride concentration for $n$-type Si (cf. also [23] for a detailed analysis of parameters and resulting morphology).

To reach a narrow distribution of pore sizes, it is necessary to induce uniform nucleation and to suppress branching. This either necessitates prepatterning by $<100 \mathrm{~nm}$ lithography, "luck," or some degree of self-organization as shown for the (macro)pores in Figure 3.

This brings us to the smallest pores, the micropores (following IUPAC). Generally, micropores grow on low-doped p-type material with ca. $1 \mu \mathrm{m} / \mathrm{min}$; crack-free growth beyond a thickness of some $\mu \mathrm{m}$ may pose a serious problem. The resulting morphology is a chaotic network of nm sized $\mathrm{Si}-$ branches with porosities up to $80 \%$ (meaning that almost any atom left is a surface atom), but we skip further details of this by far most investigated pore type and refer to two recent books $[24,25]$.
While microporous Si triggered the immense research dedicated to light-emitting Si, real devices will most likely be based on quantum structures realized with different technologies. Nevertheless, microporous layers still provide ample ground for research and may be seen as test vehicles for structures produced with more advanced technologies which are unaffordable in most laboratories. As a somewhat unwelcome feature it must be added that so far attempts to produce micropores in semiconductors other then $\mathrm{Si}$ by anodic etching were not successful, with the exception of $6 \mathrm{H}-\mathrm{SiC}$ [11]. This is not only frustrating, but also surprising, because the generally accepted formation mechanism for micropores (resting heavily on quantum wire effects) is not Si specific but should occur in any semiconductor.

\section{PORES IN OTHER SEMICONDUCTORS}

The variety of pores in III-V compounds (and to a smaller extend in $\mathrm{SiC}$ [10]) has been discussed elsewhere [8], here only some results from InP will be highlighted. InP most prominently features two different types of pores: crystallographically oriented ("crysto") and current-line-oriented ("curro") pores; which by now also have been found in GaP and Si. The former are growing at low current densities along that set of crystallographically defined $\langle 111\rangle$ directions where one "looks" from the group V to the group III atom (the so-called $\langle 111\rangle \mathrm{B}$ direction) and usually has a triangular cross section. High current densities applied to a fresh surface also first yield crysto pores, which then heavily branch (including upwards) during their growth into depth. As soon as the pore density becomes so large that further branching is impeded, the pore morphology switches synchronized into the curro mode, that is, pores are now growing along the current path (same as along electrical field lines). These pores are round and try to self-organize in a hexagonal close-packing. Several self-organizational features are encountered, most 


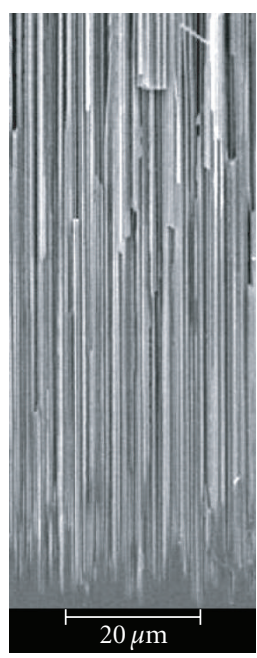

(a)

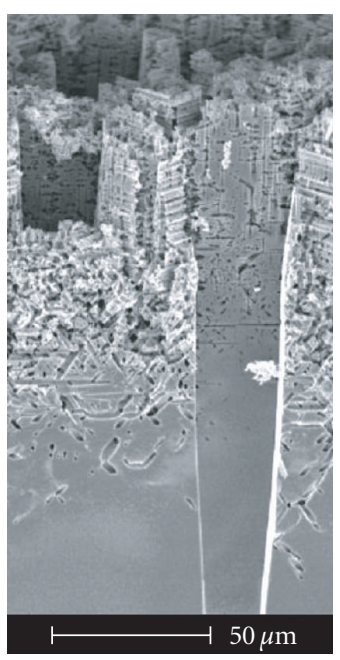

(b)

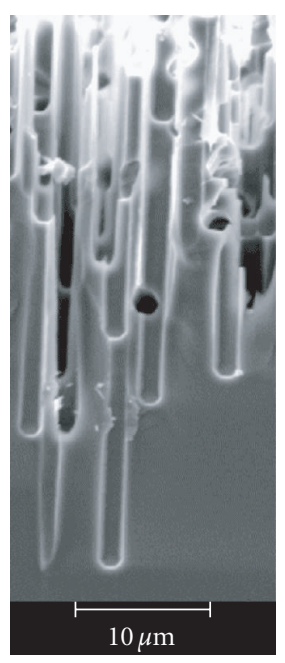

(c)

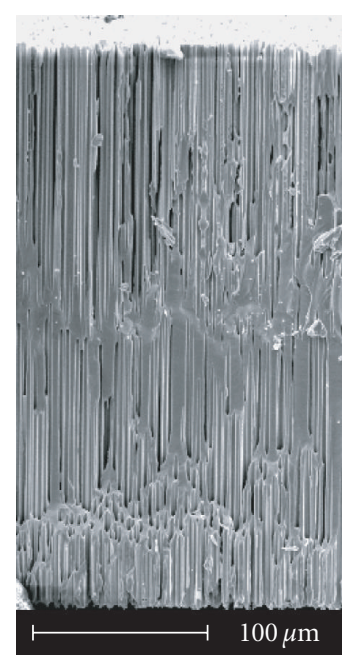

(d)

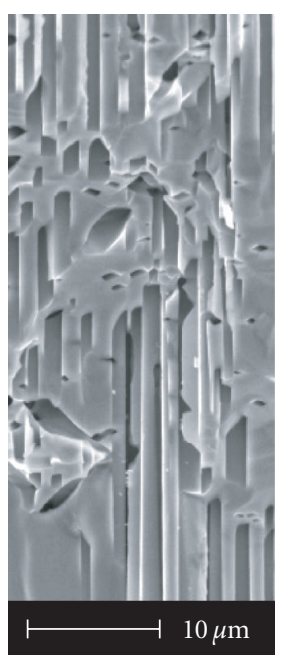

(e)

Figure 5: Pores in Ge. (a) Good sub- $\mu$ m macropores, (b) mix of several pore types, (c) macropores in $p$-type Ge, (d) Ge membrane, and (e) overlap part of membrane.

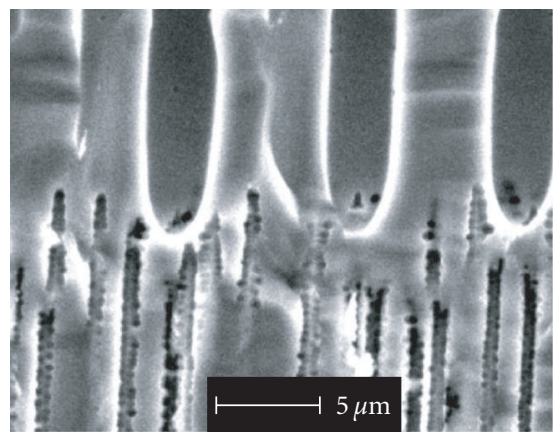

(a)

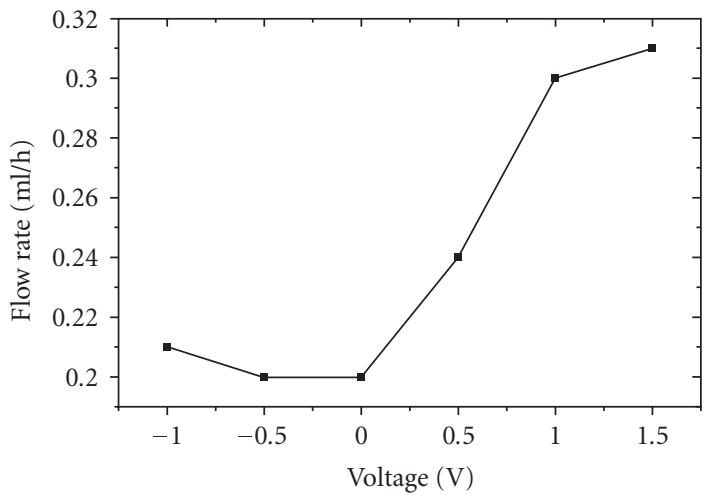

(b)

Figure 6: (a) Meso-macro membrane; for example, for fuel cells applications, (b) flow rate through a porous Ge membrane as a function of applied potential.

prominent the formation of hexagonal single pore crystal on (111) and-amazingly_-(100) substrates (cf. [22]). Induced switching between crysto and curro pores may have novel uses, as will be pointed out below.

Investigations of porous Ge began more recently [7]. While first it appeared that it was difficult to obtain pores at all [5], however by now a bewildering variety of pores has been found and some results will be reported here for the first time. Figure 5 gives a flavor of what is possible. A list of "firsts" in Ge includes (i) only other material besides Si where backside illumination can be used, however with new effects; (ii) only semiconductor besides Si where "good" pores can be produced in $p$-type material; (iii) first example for pore growth in $\langle 111\rangle$ direction for nonpolar cubic semiconductor; (iv) the only material so far with $\{110\}$ "stopping" planes. While there are many more unusual features which move Ge in the focus of efforts to arrive at a better understanding of pore formation mechanisms in general. There is one feature of porous $\mathrm{Ge}$ that is of considerable interest for potential uses: Ge exhibits the effect electrocapillarity or electrowetting, that is, its surface can be switched from hydrophobic to hydrophilic by just changing the applied potential; a feature that has been demonstrated in [7] and illustrated for our Ge membranes in Figure 6(b).

\section{MEMBRANE FABRICATION}

For many applications (especially chemical, but also optical), a porous membrane would be preferable to a layer, or is even necessary. Membrane fabrication, however, is not trivial after the electrochemical porosification step has been done. Grinding off the solid backside would be the first approach, but this process only works for rather thick and stable pore walls, that is, "big" pores. For small pores the grinding process tends to destroy the porous layer and to clog the pores with debris. 
A better method uses a wet etch-back procedure from the backside. The pore surface in this case must first be protected by an oxide and/or a nitride coating, after that the backside is exposed to a chemical etching solution (in the case of Si: $\mathrm{KOH}$ or $\mathrm{HF} / \mathrm{HNO}_{3}$ mixtures). Without any protection, the pore walls would be quickly destroyed as soon as the etching front reaches them. Especially the coating with nitride, which is for reliable protection almost unavoidable, makes this process expensive, in particular when pores with a very large aspect ratio call for very low pressure CVD.

Alternatively, a membrane can be "blasted off" the substrate by simply employing a current pulse, driving the system into the electropolishing, regime after the electrochemical pore etching is concluded. Especially for mesopore membranes, this process works pretty well and has been demonstrated for $100 \mu \mathrm{m}$ thick layers on whole $100 \mathrm{~mm}$ wafers for an industrial application.

Another possibility is a changing in the electrolyte after the pore etching to a nonfluoride containing electrolyte that only anodically oxidizes the pore tips. Under favorable circumstances the anodic oxide grows laterally until the oxide layer around pore tips connects. Afterwards the wafer is placed in a HF solution, which dissolves the oxide and disattaches the porous region. Unlike the lift-off process, this can be done outside the electrochemical cell in an optimized process to ensure that the fragile membrane remains undamaged. Both processes mentioned produce a free-standing membrane without a solid rim which is only desirable in some cases. A certain advantage is that the wafer can be reused for further membrane production.

The growth of pores from both sides of the wafer can also be used to produce membranes. Most pores do not easily intersect (i.e., Si macro + macro, micro in any combination), because they have a carrier depleted space charge region around themselves, but mesopores in Si and macropores in Ge, for example, can overcome this obstacle (cf. Figures 5(d) and 6(a)). First a deep macroporous layer is grown. Afterwards the wafer is turned around and with a second porous layer, for example, mesopores are driven in from the backside.

Depending on the planned application, the mesoporous layer can then be chemically etched-off, as mesopores are much more prone to chemical etches than macropores, oralternatively - a dual pore size membrane results. In the latter case, the mesoporous layer defines the critical dimensions for filtering applications, while the macropores help to stabilize the membrane and to controllably support the filter with the to-be-filtered solution.

\section{SOME PROPERTIES AND APPLICATIONS OF POROUS SEMICONDUCTORS}

\section{Properties}

The properties of porous semiconductor can be radically different from those of the bulk material, in particular if nanoporous materials are considered. Not only prime semiconductor parameters like conductivity, band gap, and absorption/emission of light might be quite different, but also internal symmetries/anisotropies, high-order effects and even basic chemistry. Of course, the large surface to volume ratio also plays a major role. A few examples of new properties form a long (and not yet fully established) list.

(a) More than 100-fold increase of second harmonic generation in porous $\mathrm{GaP}$, if the pore cross-section is triangular [26].

(b) Strongly increased or decreased cathodoluminescence in porous $\mathrm{GaP}$ or InP, respectively [27].

(c) Optical anisotropy (e.g., birefringence) [28] and new types of anisotropy not encountered in natural materials, if two or more sets of pores are present simultaneously [29].

(d) Novel absorption characteristic (e.g., transparent for UV while blocking larger wavelength $[25,30]$ ).

(e) Fast chemical reactions practically not observed with bulk materials; including violent explosions due to large surface to volume ratio and optimal diffusion conditions [31].

(f) Strongly decreased thermal conductivity (and concomitantly changed phonon spectra) [32].

(g) Strongly changed modes of plasmon, exciton, polariton, and so forth [25].

(h) Extreme aspect ratios (up to 10000 for InP [8]).

(i) Strongly changed conductivity, sensitive to the presence of gases or humidity [33].

\section{Applications}

The spectrum of possible applications, which exploit the special properties given above, is large; it will be grouped into several subgroups here. From each topic, one or two examples indicated in bold letters are discussed more extensively.

\section{Optics}

(i) Novel optical elements with already demonstrated capabilities are photonic crystals [34], optical short-pass filters [25, 30], as well as Bragg and Rugate mirrors obtained by modulating porosity with depth [35].

(ii) Emerging applications only partially demonstrated include nonlinear elements (e.g., strong frequency doubling in porous GaP [26] and $\mathrm{Si}$ [36]) including phase matching, integrated waveguides [37], and novel anisotropic (e.g., birefringent) materials [29].

Structures with the potential of being used as integrated waveguides in InP rely on the possibility to abruptly switch from crysto to curro pores; Figure 7 shows an example. The easily induced porosity changes are coupled to a change in the refractive index suitable for waveguiding. These structures are easy to make (in contrast to conventional techniques), if all their properties (including, e.g., damping) are suitable for optoelectronic devices remains to be seen, but their usefulness for optical gas sensing is obvious. Optical gas and fluid sensing rely on sending light through a space filled with the gas or fluid and detecting changes of the spectrum at 


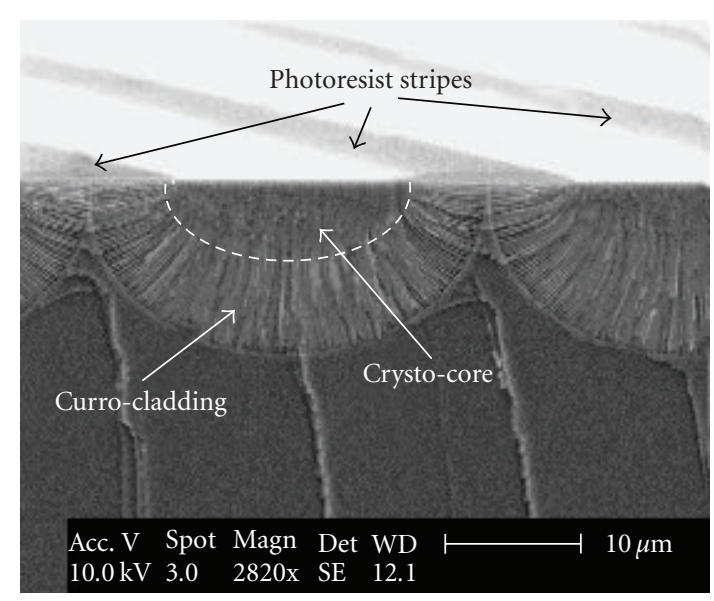

FIGURE 7: SEM cross-section of porous InP waveguides suitable for, for example, optical gas analysis: the wafer was coated with stripes of photo resist prior to electrochemical etching leading to semicylindrical pore structures. First crysto pores (low porosity) form a high index core surrounded by a low index curro cladding [37].

the end. One major problem is to confine the light on small cross section so that small quantities of substances can be analyzed. Also the signal-to-noise rises if the light is focused. Normal light waveguides are solid, that is, the analyzed substance cannot enter. A porous waveguide circumvents this problem without abandoning the waveguide properties.

A cubic crystal full of pores extending in several equivalent crystallographic directions no longer has a cubic symmetry from the viewpoint of light with a wavelength larger than the pore geometry. Indeed, (110)-oriented $\mathrm{Si}$, with two sets of mesopores extending along the $\langle 100\rangle$ directions, shows a pronounced birefringence [28] that can be much stronger than in natural crystals. Recently, this effect has been recalculated with considerable accuracy; in addition the theory has been extended to cover other pore geometries. Completely new anisotropic behavior was predicted for certain pore geometries that might be producible in GaAs or InP [29] (cf. Figure 8). Filling of the pores with a metal exhibiting strong plasmonic resonances (e.g., for Ag) is expected to further enhance these optical anisotropies [38].

\section{Mechanics}

(i) Porous layers are generally useful for MEMS applications, most simply as sacrificial layers, but also as a kind of dielectric layer or as thermal insulation. A large-scale effort intends to use macroporous Si electrodes as well as microporous Si electrodes for microfuel cells [39-41].

(ii) The extremely low thermal conductivity has been employed for ultrasonic generation [42].

(iii) In one of the so far very few large-scale application, canon uses porous layers as a kind of "zipper" during the fabrication of silicon on insulator (SOI) wafers [43]. (iv) Porous layers are as buffer layers for demanding epitaxial processes, for example, $\mathrm{GaN}$ on $\mathrm{SiC}$ [44].

(v) Porous layers serve as templates for nanorods or nanotubes of many different kinds [45].

(vi) Microporous Si is a key ingredient for a high-power explosive [31].

If the pores of microporous Si are filled with an oxidizer, owing to the huge surface area, an amazingly potent explosive results. Research is under way to explore this (accidentally discovered) property for applications like an airbag firing device. Exotic as it appears, this application might be closer to a product than many sensor developments.

\section{Chemical sensing}

Generally, several basic effects can be used for chemical sensing (i) functionalized pore surfaces are sensitive to certain substances only, binding the analyte changes directly measurable properties like photo luminescence, (spectral) reflectivity, or conductivity/impedance. (ii) Special optical elements made from porous materials, most notably Bragg reflectors and resonators, change their key property (e.g., the wavelength of reflections/diffraction under a certain angle) in easily measurable ways. (iii) Specific properties of a photonic crystal made from porous materials can be used, for example, a very small effective light propagation speed which allows to miniaturize conventional optical gas spectrometers [46].

Smart dust from the Sailor group in San Diego [47] carries the Bragg reflector principle to the extreme. Several sets of mesoporous silicon rugate filters are functionalized for different protein groups (i.e., one mirror color equals one specific protein) and then ground to dust which can be blown into the atmosphere or buildings. The dust particles, if exposed to the test substance or gas, will bind the protein to be detected and then change their reflective behavior to something that can be remotely detected with a LASER.

\section{Biotech uses}

(i) Filters for proteins and other particles. Due to the low dispersion in pore size, electrochemically etched mesoand macropores represent optimal candidates for filtering application with a high-size selectivity, for example, filtering of protein mixtures. As discussed in the "Membrane fabrication" section of this article, a combination of large macropores and smaller mesopores (cf. Figure 6(a)) offers a unique combination of mechanical stability, production ease, and filtering capabilities.

(ii) Very regular arrays of macropores in Si are already used for biochip applications (DNA); and the technology is easily scaled to much smaller sizes if needed [25].

(iii) Bio MEMS applications are emerging, for example, utilizing porous structures as templates for living cells on porous substrates [48].

(iv) Microrefinery and reactors. While some work has been done along this direction [49], this is still more a vision 


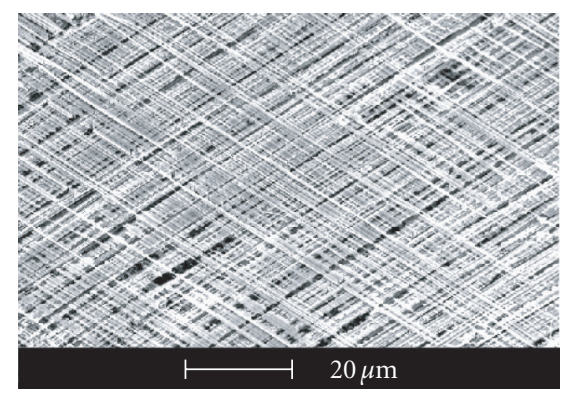

(a)

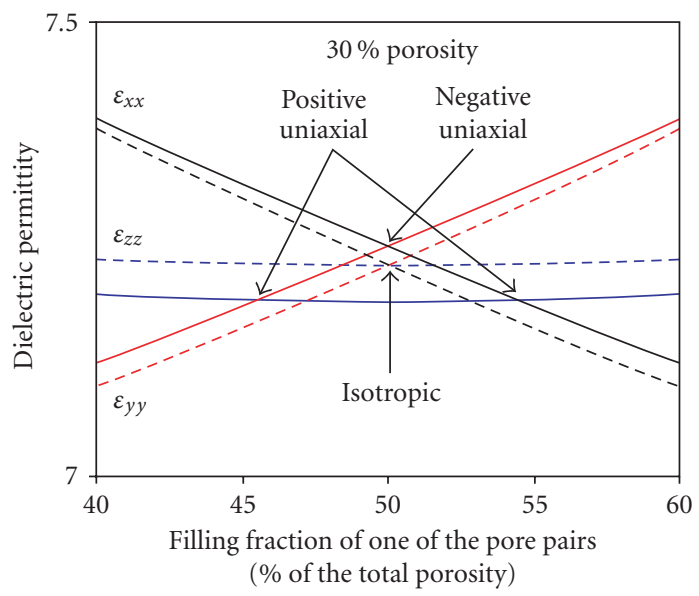

(b)

Figure 8: (a) Two $\langle 111\rangle$ sets of pores in (100) GaAs. (b) Numerically calculated dielectric tensor elements of the porous GaAs layer shown in (a) as a function of the relative filling fraction of one of the pore lattices; the overall porosity of the sample is $30 \%$. Dashed lines: pores with circular cross-section, solid lines: triangular cross-sections of crystallographic pores in GaAs. If the relative porosities of the two sets of pores are changed, novel properties result.

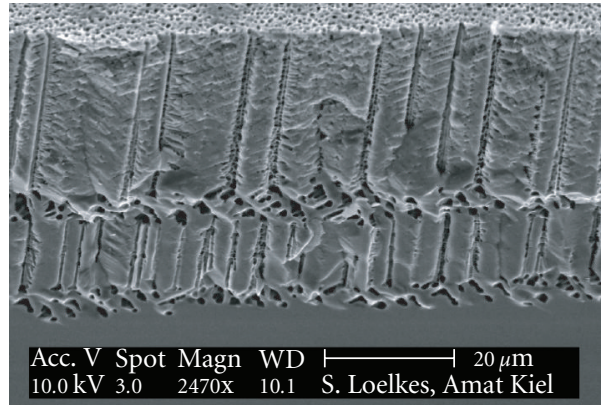

(a)

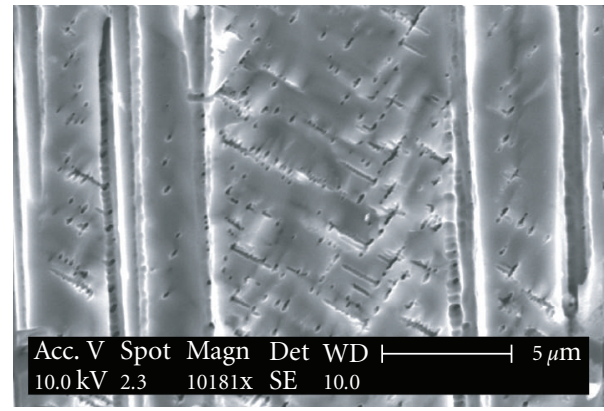

(b)

FIGURE 9: Sequential combination of different pore directions and densities. (a) Si pores switching between $\langle 111\rangle$ directions and $\langle 113\rangle$ directions. (b) Combination of meso- and macropores.

than a fact. With certain techniques it is possible to interconnect straight pores, while still exercising some control over the pore geometry; Figure 9 shows examples. In Figure 9(a) the pores grow along $\langle 111\rangle$, but change to $\langle 113\rangle$ (with three equivalent directions) and back if the voltages are cycled in a suitable way. The "fast etching" modes for Si (cf. Figure 3) and also for InP (cf. Figure 1(c)) offer similar morphologies superimposing crysto and curro pores. Figure 9(b) shows macropores interconnected by mesopores (usually considered a nuisance). All these structures resemble biological tissue optimized for processing of complex liquids, and it is tempting to speculate about applications as indicated above.

\section{ACKNOWLEDGMENTS}

M. Kemell, M. Christophersen, E. Ossei-Wusu, C. Fang, S. Langa supplied data, and AMD, MEMC; Siltronic and
Infineon supplied wafers or processes; this is gratefully acknowledged. Parts of this work were supported by the DFG.

\section{REFERENCES}

[1] L. T. Canham, "Silicon quantum wire array fabrication by electrochemical and chemical dissolution of wafers," Applied Physics Letters, vol. 57, no. 10, pp. 1046-1048, 1990.

[2] V. Lehmann and U. Gösele, "Porous silicon formation: a quantum wire effect," Applied Physics Letters, vol. 58, no. 8, pp. 856858, 1991.

[3] H. Föll, M. Christophersen, J. Carstensen, and G. Hasse, "Formation and application of porous silicon," Materials Science and Engineering: R: Reports, vol. 39, no. 4, pp. 93-141, 2002.

[4] A. Uhlir, "Electrolytic shaping of germanium and silicon," Bell System Technical Journal, vol. 35, pp. 333-347, 1956.

[5] K. Choi and J. M. Buriak, "Hydrogermylation of alkenes and alkynes on hydride-terminated Ge(100) surfaces," Langmuir, vol. 16, no. 20, pp. 7737-7741, 2000.

[6] S. Langa, M. Christophersen, J. Carstensen, I. M. Tiginyanu, and H. Föll, "Electrochemical pore etching in Ge," Physica Status Solidi (A), vol. 195, no. 3, pp. R4-R6, 2003. 
[7] C. Fang, H. Föll, and J. Carstensen, "Electrochemical pore etching in germanium," Journal of Electroanalytical Chemistry, vol. 589, no. 2, pp. 259-288, 2006.

[8] H. Föll, S. Langa, J. Carstensen, M. Christophersen, and I. M. Tiginyanu, "Pores in III-V semiconductors," Advanced Materials, vol. 15, no. 3, pp. 183-198, 2003.

[9] M. Mynbaeva, A. Titkov, A. Kryzhanovski, et al., "Strain relaxation in GaN layers grown on porous GaN sublayers," MRS Internet Journal of Nitride Semiconductor Research, vol. 4, p. 14, 1999.

[10] S. Zangooie, J. A. Woollam, and H. Arwin, "Self-organization in porous 6H-SiC," Journal of Materials Research, vol. 15, no. 9, pp. 1860-1863, 2000.

[11] J. S. Shor, L. Bemis, A. D. Kurtz, et al., "Characterization of nanocrystallites in porous p-type 6H-SiC," Journal of Applied Physics, vol. 76, no. 7, pp. 4045-4049, 1994.

[12] E. Monaico, V. V. Ursaki, A. Urbieta, et al., "Porosity-induced gain of luminescence in CdSe," Semiconductor Science and Technology, vol. 19, no. 12, pp. L121-L123, 2004.

[13] L. T. Canham, A. Nassiopoulou, and V. Parkhutik, "Proceedings of the 3rd international conference porous semiconductors-science and technology," Physica Status Solidi (A), vol. 197, no. 1, 2003.

[14] V. Lehmann and H. Föll, "Formation mechanism and properties of electrochemically etched trenches in n-type silicon," Journal of the Electrochemical Society, vol. 137, no. 2, pp. 653659, 1990.

[15] Y. Shishkin, Y. Ke, R. P. Devaty, and W. J. Choyke, to appear in Physica Status Solidi (A).

[16] C. Fang, S. Langa, L. Jiang, J. Carstensen, E. Foca, and H. Föll, Electrochemical pore etching in Germanium, 3.8 (2005).

[17] V. Lehmann and S. Rönnebeck, "The physics of macropore formation in low-doped p-type silicon," Journal of the Electrochemical Society, vol. 146, no. 8, pp. 2968-2975, 1999.

[18] J. Carstensen, R. Prange, and H. Föll, "A model for currentvoltage oscillations at the silicon electrode and comparison with experimental results," Journal of the Electrochemical Society, vol. 146, no. 3, pp. 1134-1140, 1999.

[19] ET\&TE Etch and Technology GmbH, http://www.et-te.com.

[20] J. E. A. M. van den Meerakker, R. J. G. Elfrink, F. Roozeboom, and J. F. C. M. Verhoeven, "Etching of deep macropores in 6 in. Si wafers," Journal of The Electrochemical Society, vol. 147, no. 7, pp. 2757-2761, 2000.

[21] S. Frey, M. Kemell, J. Carstensen, S. Langa, and H. Föll, "Fast pore etching," Physica Status Solidi (A), vol. 202, no. 8, pp. 1369-1373, 2005.

[22] S. Langa, I. M. Tiginyanu, J. Carstensen, M. Christophersen, and H. Föll, "Self-organized growth of single crystals of nanopores," Applied Physics Letters, vol. 82, no. 2, pp. 278-280, 2003.

[23] V. Lehmann, R. Stengl, and A. Luigart, "On the morphology and the electrochemical formation mechanism of mesoporous silicon," Materials Science and Engineering B, vol. 69-70, pp. 11-22, 2000.

[24] X. G. Zhang, Electrochemistry of Silicon and Its Oxide, Kluwer Academic/Plenum, New York, NY, USA, 2001.

[25] V. Lehmann, Electrochemistry of Silicon, Wiley-VCH, Weinheim, Germany, 2002.

[26] I. M. Tiginyanu, I. V. Kravetsky, S. Langa, G. Marowsky, J. Monecke, and H. Föll, "Porous III-V compounds as nonlinear optical materials,” Physica Status Solidi (A), vol. 197, no. 2, pp. 549-555, 2003.

[27] I. M. Tiginyanu, S. Langa, L. Sirbu, E. Monaico, M. A. StevensKalceff, and H. Föll, "Cathodoluminescence microanalysis of porous GaP and InP structures," The European Physical Journal: Applied Physics, vol. 27, no. 1-3, pp. 81-84, 2004.

[28] D. Kovalev, G. Polisski, J. Diener, et al., "Strong in-plane birefringence of spatially nanostructured silicon," Applied Physics Letters, vol. 78, no. 7, pp. 916-918, 2001.

[29] V. Kochergin, M. Christophersen, and P. R. Swinehart, "Macroporous silicon UV filters for space and terrestrial environments," in Photonics for Space Environments IX, vol. 5554 of Proceedings of SPIE, pp. 223-234, Denver, Colo, USA, August 2004.

[30] V. Kochergin, Omnidirectional Optical Filters, Kluwer Academic, Boston, Mass, USA, 2003.

[31] D. Kovalev, V. Yu. Timoshenko, N. Künzner, E. Gross, and F. Koch, "Strong explosive interaction of hydrogenated porous silicon with oxygen at cryogenic temperatures," Physical Review Letters, vol. 87, no. 6, Article ID 068301, 4 pages, 2001.

[32] G. Kaltsas and A. G. Nassiopoulou, "Novel C-MOS compatible monolithic silicon gas flow sensor with porous silicon thermal isolation," Sensors and Actuators A: Physical, vol. 76, no. 1-3, pp. 133-138, 1999.

[33] C. J. Oton, L. Pancheri, Z. Gaburro, et al., "Multiparametric porous silicon gas sensors with improved quality and sensitivity," Physica Status Solidi (A), vol. 197, no. 2, pp. 523-527, 2003.

[34] K. Busch, S. Lölkes, R. B. Wehrspohn, and H. Föll, Eds., Photonic Crystals: Advances in Design, Fabrication, and Characterization, Wiley-VCH, Weinheim, Germany, 2004.

[35] C. Mazzoleni and L. Pavesi, "Application to optical components of dielectric porous silicon multilayers," Applied Physics Letters, vol. 67, no. 20, pp. 2983-2985, 1995.

[36] T. V. Dolgova, A. I. Maidykovski, M. G. Martemyanov, et al., "Giant optical second-harmonic generation in single and coupled microcavities formed from one-dimensional photonic crystals," Journal of the Optical Society of America B: Optical Physics, vol. 19, no. 9, pp. 2129-2140, 2002.

[37] S. Langa, S. Frey, J. Carstensen, et al., "Waveguide structures based on porous indium phosphide," Electrochemical and Solid-State Letters, vol. 8, no. 2, pp. C30-C32, 2005.

[38] V. Kochergin, M. Christophersen, and P. R. Swinehart, "Macroporous silicon-based polarization components," in Nanoengineering: Fabrication, Properties, Optics, and Devices, vol. 5515 of Proceedings of SPIE, pp. 132-141, Denver, Colo, USA, August 2004.

[39] L. Ohlsen, to appear in Physica Status Solidi (A).

[40] S. Gold, K.-L. Chu, C. Lu, M. A. Shannon, and R. I. Masel, "Acid loaded porous silicon as a proton exchange membrane for micro-fuel cells," Journal of Power Sources, vol. 135, no. 1-2, pp. 198-203, 2004.

[41] T. Pichonat and B. Gauthier-Manuel, "Development of porous silicon-based miniature fuel cells," Journal of Micromechanics and Microengineering, vol. 15, no. 9, pp. S179-S184, 2005.

[42] H. Shinoda, T. Nakajima, K. Ueno, and N. Koshida, "Thermally induced ultrasonic emission from porous silicon," $\mathrm{Na}$ ture, vol. 400, no. 6747, pp. 853-855, 1999.

[43] T. Yonehara, "BESOI with porous silicon: ELTRAN," in Properties of Porous Silicon, L. T. Canham, Ed., IEE-Books, London, UK, 1997.

[44] C. K. Inoki, T. S. Kuan, C. D. Lee, et al., "Growth of GaN on porous $\mathrm{SiC}$ and GaN substrates," Journal of Electronic Materials, vol. 32, no. 8, pp. 855-860, 2003.

[45] M. Steinhart, Z. Jia, A. K. Schaper, R. B. Wehrspohn, U. Gösele, and J. H. Wendorff, "Palladium nanotubes with tailored wall morphologies," Advanced Materials, vol. 15, no. 9, pp. 706709, 2003. 
[46] R. B. Wehrspohn, S. L. Schweizer, J. Schilling, T. Geppert, C. Jamois, and R. Glatthaar, "Application of photonic crystals for gas detection and sensing," in Photonic Crystals: Advances in Design, Fabrication, and Characterization, K. Busch, S. Lölkes, R. Wehrspohn, and H. Föll, Eds., Wiley-VCH, Weinheim, Germany, 2004.

[47] F. Cunin, T. A. Schmedake, J. R. Link, et al., "Biomolecular screening with encoded porous-silicon photonic crystals," $\mathrm{Na}$ ture Materials, vol. 1, no. 1, pp. 39-41, 2002.

[48] S. C. Bayliss, P. J. Harris, L. D. Buckberry, and C. Rousseau, "Phosphate and cell growth on nanostructured semiconductors," Journal of Materials Science Letters, vol. 16, no. 9, pp. 737-740, 1997.

[49] T. Laurell, J. Drott, L. Rosengren, and K. Lindström, "Enhanced enzyme activity in silicon integrated enzyme reactors utilizing porous silicon as the coupling matrix," Sensors and Actuators B: Chemical, vol. 31, no. 3, pp. 161-166, 1996. 

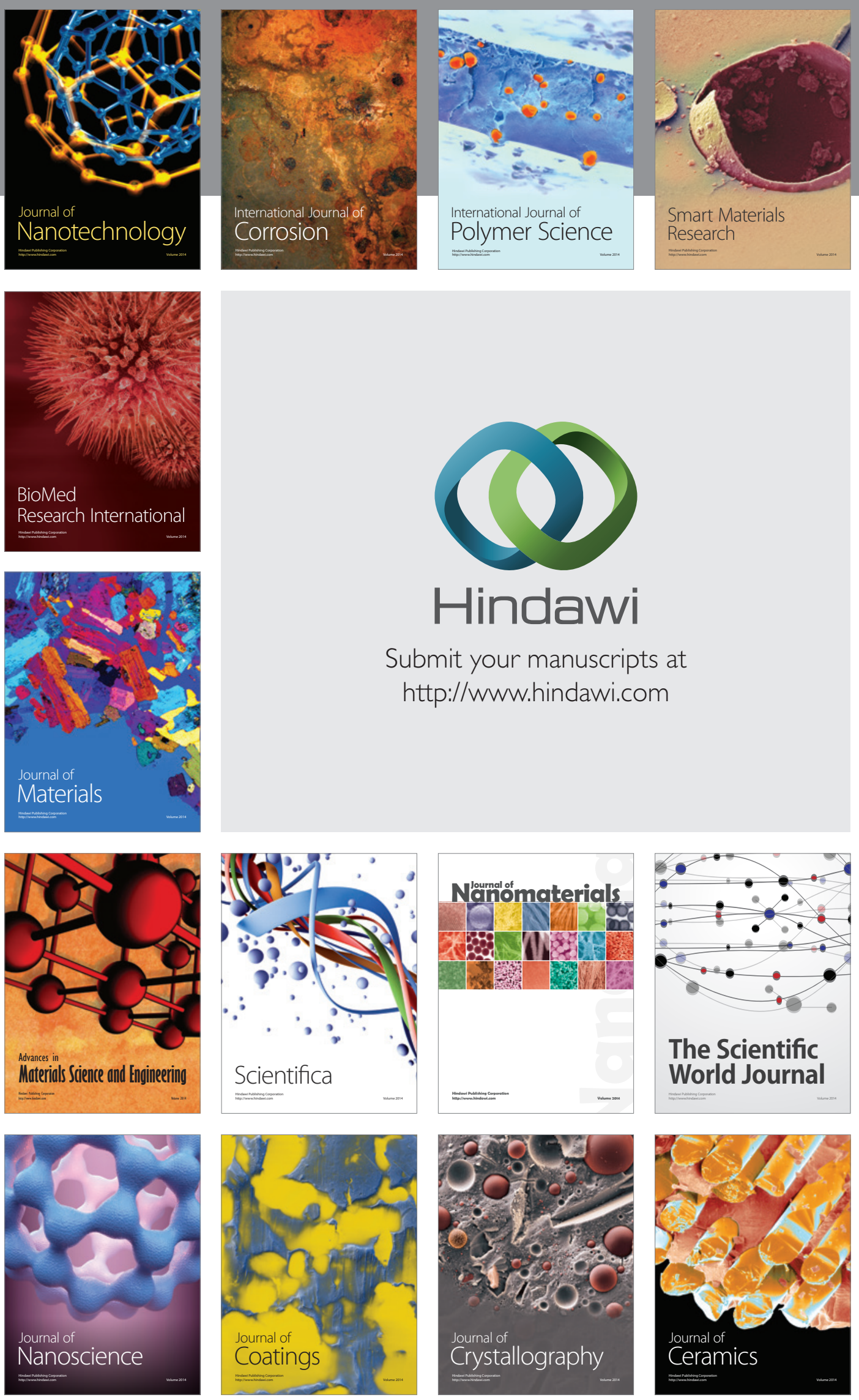

The Scientific World Journal

Submit your manuscripts at

http://www.hindawi.com

\section{World Journal}

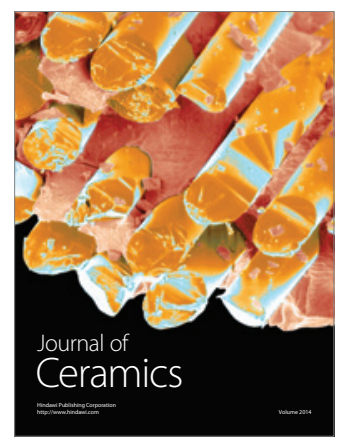

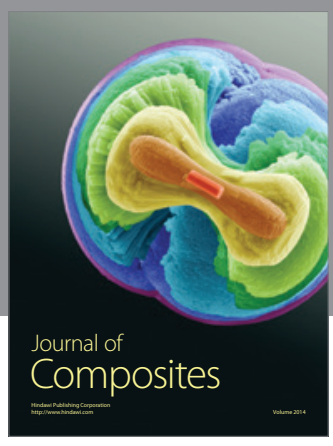
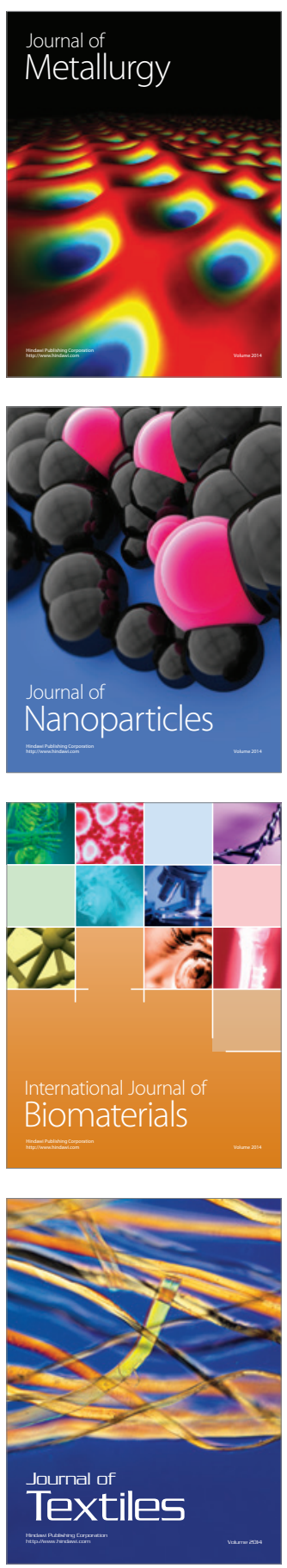